\title{
FACTORES ASOCIADOS AL USO REGULAR DE FUENTES DE INFORMACIÓN EN ESTUDIANTES DE MEDICINA DE CUATRO CIUDADES DEL PERÚ
}

\author{
Christian R. Mejía ${ }^{1, a}$, Mario J. Valladares-Garrido, ${ }^{2, b}$, Aldo Luyo-Rivass,a, Danai Valladares-Garrido,a, \\ Lincolth Talledo-Ulfe ${ }^{2, b}$, Martín A. Vilela-Estrada ${ }^{4, b}$, Mary M. Araujo Chumacero ${ }^{5, b}$, Red GIS Peru*
}

RESUMEN

Objetivos. Determinar los factores asociados al uso regular de fuentes de información en estudiantes de Medicina de cuatro ciudades de Perú. Materiales y métodos. Estudio transversal analítico, se encuestó a estudiantes de Medicina de cuatro ciudades del Perú, recopilando información del uso de 14 fuentes de información y otras variables educativas e informáticas. Se definió uso frecuente de la fuente de información si accedía a ella mínimo una vez a la semana. Se obtuvieron los valores $p$ mediante modelos lineales generalizados ajustando por la sede de cada encuestado. Resultados. Se encuestaron 2300 estudiantes con una mediana de edad de 21 años, el $53 \%$ fueron mujeres. El recibir una capacitación para el uso de las fuentes incrementó el uso en doce de las bases consultadas, no en SciELO $(p=0,053)$ ni en la biblioteca universitaria $(p=0,509)$. Cuando se añadió el ajuste por poseer una laptop/netbook se mantuvieron dichas asociaciones. Al ajustar también por poseer un smartphone se perdió la asociación con la base BVS Perú $(p=0,067)$, lo mismo ocurrió al hacer el último ajuste, si había realizado alguna actividad de investigación. Conclusiones. El uso frecuente de las fuentes de la información está asociado con haber recibido capacitación, realizar investigación y el uso de las tecnologías de la información y comunicación. Esto debe ser tomado en cuenta en programas de capacitación y mejora continua en el pre y posgrado.

Palabras clave: Internet; Bibliografía; Bases de datos de citas (fuente DeCS BIREME).

\section{FACTORS ASSOCIATED WITH THE REGULAR USE OF SOURCES OF INFORMATION BY MEDICAL STUDENTS FROM FOUR CITIES OF PERU}

\begin{abstract}
Objetives. To determine the factors associated with regular use of sources of information by medical students in four cities in Peru. Materials and methods. In this cross-sectional study, medical students were surveyed in four cities of Peru, gathering information on the use of 14 sources of information and other educational and computer variables. Frequent use of the information source was defined if the respondent reported that they access an information source at least once a week. P values were obtained by generalized linear models adjusted for each respondent site. Results. 2,300 students were surveyed. The median age was 21 years and $53 \%$ were women. Having received training in the use of sources increased the use in twelve of the consulted bases, not in SciELO $(p=0.053)$ or in the university library $(p=0.509)$. When adjusting for owning a laptop/netbook, these associations remained. After also adjusting for owning a smartphone the association was lost with the BVS Peru database $(p=0.067)$. The association was also lost after making the final adjustment, if the respondent had carried out any research activities. Conclusions. The frequent use of sources of information is associated with having received training, conducting research and use of information technologies and communication. This should be taken into account in training programs and continuous improvement in undergraduate education.
\end{abstract}

Key words: Internet; Bibliography; Citation databases (source: MeSH NLM).

\footnotetext{
Escuela de Medicina, Universidad Peruana de Ciencias Aplicadas. Lima, Perú.

Escuela de Medicina, Universidad César Vallejo. Piura, Perú.

Escuela de Posgrado de Medicina, Universidad San Martín de Porres. Lima, Perú

Escuela de Medicina, Universidad Privada Antenor Orrego. Piura, Perú.

Escuela de Medicina, Universidad Nacional de Piura. Piura, Perú.

* Grupo de Investigación de las SOCEM.

a Médico cirujano; ${ }^{\text {b }}$ estudiante de Medicina.

Recibido: : 15-03-15 Aprobado: 27-05-15
}

Citar como: Mejia CR, Valladares-Garrido MJ, Luyo-Rivas A, Valladares-Garrido D, Talledo-Ulfe L, Vilela-Estrada MA, et al. Factores asociados al uso regular de fuentes de información en estudiantes de Medicina de cuatro ciudades del Perú. Rev Peru Med Exp Salud Publica. 2015;32(2):230-6. 


\section{INTRODUCCIÓN}

El Centro Cochrane Iberoamericano tituló a una publicación de 1972 "Efectividad y eficiencia: reflexiones aleatorias sobre los servicios de salud," en la que constató que gran parte de la práctica clínica habitual carecía de evidencia sobre su efectividad (1). Después de cuarenta años es todavía uno de los problemas generales de los servicios de salud: la falta de evidencia para la toma de decisiones ${ }^{(2)}$. Internet, como uno de los adelantos tecnológicos del último siglo, es indispensable dentro de la práctica médica e influye en el manejo e intercambio de información ${ }^{(3)}$. Pudiendo considerarse como una "negligencia" que los médicos no utilicen las herramientas necesarias para capacitarse en cuanto a búsqueda confiable de información; es así que, en el futuro el no dominar estas herramientas informáticas ha de ser considerado como una incapacidad, tanto como actualmente se considera al no saber leer o escribir ${ }^{(4)}$.

Es en este campo que el uso de las tecnologías de información y comunicación (TIC) ha demostrado ser de gran relevancia, para el avance científico y tecnológico; generando cambios claves en el área de la medicina, en prestación de servicios de salud, formación de recursos humanos -permitiendo desarrollar de una forma eficaz su capacitación y actualización continua- e investigación ${ }^{(5)}$. Existen algunos reportes en Latinoamérica que refieren que la gran mayoría de estudiantes de Medicina saben manejar Internet, pero con desconocimiento acerca de cuáles son las fuentes confiables ${ }^{(6)}$. Así también, los médicos tienen poco conocimiento sobre el uso y las ventajas de las TIC, pese a la gran importancia que esto cobra para la actual práctica profesional ${ }^{(3,7)}$.

En el Perú es escasa la cantidad de reportes que muestren el uso de fuentes y TIC en estudiantes de Medicina y en médicos ${ }^{(2,7-9)}$. Es por lo mencionado que se realizó un estudio para determinar los factores asociados al uso regular de fuentes de información en estudiantes de Medicina de cuatro ciudades.

\section{MATERIALES Y MÉTODOS}

\section{DISEÑO Y POBLACIÓN DE ESTUDIO}

Se realizó un estudio multicéntrico observacional de tipo transversal analítico, en el periodo julio de 2013 hasta mayo de 2014, en estudiantes de Medicina de universidades de Piura, Cusco, Arequipa y Lima. En cada ciudad se entrevistó a estudiantes de una universidad particular y una universidad nacional (excepto en Piura que se entrevistó a estudiantes de la universidad nacional y de dos universidades particulares), estas sedes y universidades fueron elegidas por conveniencia; por el hecho de ser las ciudades más grandes del Perú.

El muestreo fue no probabilístico, se incluyeron a estudiantes de Medicina que voluntariamente aceptaron participar del estudio (la tasa de rechazo fue menor al 3\%) y que estuvieron matriculados hasta el penúltimo año de la carrera (no internos de Medicina), se excluyeron a los que no respondieron de manera adecuada al cuestionario o que no completaron las variables de interés (uso de fuentes de la información y recursos tecnológicos).

\section{VARIABLES}

La variable principal fue el uso de fuentes de información para literatura médica científica: biblioteca física, PubMed, Biblioteca Cochrane, UpToDate, HINARI, SciELO, LILACS, LIPECS, BVS Perú, Proquest, EBSCO, OVID, Embase y Wholis. Cada opción tuvo seis alternativas de respuesta: no la conoce, nunca la usa, la usa una vez al año, la usa una vez al mes, la usa una vez a la semana o la usa a diario. Para el análisis estadístico bivariado y multivariado se dicotomizó según su frecuencia de uso, se definió uso frecuente de la fuente de información si accedía a ella cuando menos una vez a la semana, siendo las otras opciones (una vez al mes, una vez al año, no la conozco y no la uso) consideradas como no uso frecuente.

Se midieron variables socioeducativas (sexo, edad, manejo del idioma inglés, presentación de un trabajo de investigación en algún evento científico médico o estudiantil, publicación de alguna investigación científica en alguna revista indizada y la intención de titularse como médico por la modalidad de sustentación de tesis), el tener equipos informáticos y el uso de tecnología relacionada (computadora fija, laptop/netbook, uso de Internet fijo/en casa, uso de Internet inalámbrico, uso de teléfono móvil, poseer un smartphone o palm). Además, si en el último año: usó Internet, consultó a revistas médicas peruanas, participó en foros virtuales biomédicos y si recibió capacitación para el uso de base de datos/fuentes de información (definida como la recepción de algún curso para el uso y/o manejo de las fuentes de la información).

Todo esto fue preguntado en una ficha de recolección de datos autoaplicada, usada previamente en una población similar en la ciudad de Lima Metropolitana; validada en su forma y fondo antes de ser usada.

\section{PROCEDIMIENTOS}

Para la ejecución del estudio se solicitó el permiso respectivo a las sedes del encuestado. Posterior a la 
aprobación de cada docente (para la aplicación en los salones), se les proporcionó la encuesta al inicio o al finalizar el desarrollo de una clase en particular. Se obtuvo el consentimiento verbal de los encuestados para participar en el estudio. La encuesta fue anónima, los datos fueron manejados como confidenciales y se asignó un código a cada una. Aquellas que no se completaron adecuadamente o que tuvieron patrones repetitivos fueron excluidas (menos del 1\%), luego se procedió a la doble digitación en el programa Microsoft Excel (versión 2010 para Windows). Finalmente, se hizo una limpieza de la base de datos, previa al análisis estadístico, y se eliminó 38 encuestas que no tuvieron los datos principales requeridos para el análisis.

\section{ANÁLISIS ESTADÍSTICO}

Los análisis fueron realizados con el programa Stata v. 11,1 (StataCorp LP, College Station, TX, USA). Para el análisis descriptivo de las variables numéricas se evaluó los supuestos de normalidad, usando la prueba de Shapiro Wilk, según eso, se describió la mejor medida de tendencia central y dispersión, para las variables categóricas se describieron las frecuencias y porcentajes.

Se realizó el análisis bivariado y multivariado usando como variable dependiente el uso frecuente de cada una de las fuentes de la información. Se reportó los valores $p$ obtenidos del chi cuadrado (para la asociación entre el uso de tecnologías de la información según cada sede) y de los modelos lineales generalizados (GLM por sus siglas en inglés) usando la familia Poisson, la función de enlace log, modelos robustos y se tomó en cuenta como cluster a cada sede del encuestado (bajo el supuesto que los grupos difieren según sus enseñanzas, metodologías de estudio y currículas). Se consideró un $p<0,05$ como significativo.

\section{CONSIDERACIONES ÉTICAS}

El proyecto fue aprobado por el Comité de Ética del Hospital Nacional Docente Madre Niño (Código CEI: EX15011). Se respetó en todo momento la privacidad de los encuestados, las encuestas fueron anónimas y no se pidieron datos de identificación.

\section{RESULTADOS}

De los casi 3800 estudiantes de las universidades encuestadas, 2300 respondieron la encuesta; el 52,9\% (1216) fueron mujeres, con una mediana de edad de 21 años (rango intercuartílico: 16-38 años). El 60,2\% (1384) de los estudiantes fueron de universidades particulares.
Tabla 1. Características socioeducativas de los estudiantes de Medicina de cuatro ciudades del Perú

\begin{tabular}{|c|c|c|}
\hline Variable & $\mathbf{n}$ & $(\%)$ \\
\hline \multicolumn{3}{|l|}{ Sexo } \\
\hline Masculino & 1084 & $(47,1)$ \\
\hline Femenino & 1216 & $(52,9)$ \\
\hline Edad (años)* & 21 & $(16-38)$ \\
\hline \multicolumn{3}{|l|}{ Ciudad } \\
\hline Piura & 749 & $(32,6)$ \\
\hline Cusco & 720 & $(31,3)$ \\
\hline Lima & 520 & $(22,6)$ \\
\hline Arequipa & 311 & $(13,5)$ \\
\hline \multicolumn{3}{|l|}{ Universidad } \\
\hline Nacional & 916 & $(39,8)$ \\
\hline Particular & 1384 & $(60,2)$ \\
\hline \multicolumn{3}{|l|}{ Se comunica en inglés } \\
\hline No & 277 & $(12,1)$ \\
\hline Sí & 2004 & $(87,9)$ \\
\hline Nivel básico & 878 & $(43,8)$ \\
\hline Nivel intermedio & 861 & $(43,0)$ \\
\hline Nivel avanzado & 265 & $(13,2)$ \\
\hline Realiza investigación & 309 & $(13,6)$ \\
\hline Ha publicado** & 82 & $(3,7)$ \\
\hline Planea hacer tesis & 64 & $(3,7)$ \\
\hline
\end{tabular}

*Mediana y rango intercuartílico. **En alguna revista científica.

Los niveles básico e intermedio del idioma inglés fueron los más comunes (44 y 43\%, respectivamente). El 13,6\% había realizado alguna investigación científica y menos del $4 \%$ habían publicado un artículo científico o planeaba graduarse por la sustentación de tesis (Tabla 1).

La mediana de horas que usa Internet a la semana fue 8 (rango intercuartílico: 5-14). Según el uso de dispositivos y tecnología, la mayoría manifestó tener un teléfono móvil (94\%), una computadora fija (90\%), Internet fijo en casa $(83 \%)$, laptop/netbook $(73 \%)$, Internet inalámbrico $(66 \%)$ y un smartphone (57\%); muy pocos usaban una palm ( $9 \%)$. La Tabla 2 muestra el porcentaje de uso de cada uno según la sede del encuestado.

Tabla 2. Porcentaje de uso de equipos y dispositivos de tecnología según ciudad

\begin{tabular}{lcccccc}
\hline \multicolumn{1}{c}{ Uso de } & Lima & Piura & Arequipa & Cusco & Valor $\boldsymbol{p}$ \\
\hline Teléfono móvil & 89 & 97 & 96 & 93 & $<0,001$ \\
Computadora fija & 91 & 84 & 96 & 92 & $<0,001$ \\
Internet fijo & 89 & 83 & 91 & 74 & $<0,001$ \\
Laptop/netbook & 66 & 77 & 65 & 75 & $<0,001$ \\
Internet inalámbrico & 69 & 75 & 54 & 58 & $<0,001$ \\
Smartphone & 66 & 71 & 44 & 42 & $<0,001$ \\
Palm & 17 & 12 & 3 & 3 & $<0,001$ \\
\hline
\end{tabular}

Valor $p$ obtenido con la prueba estadística chi cuadrado. 


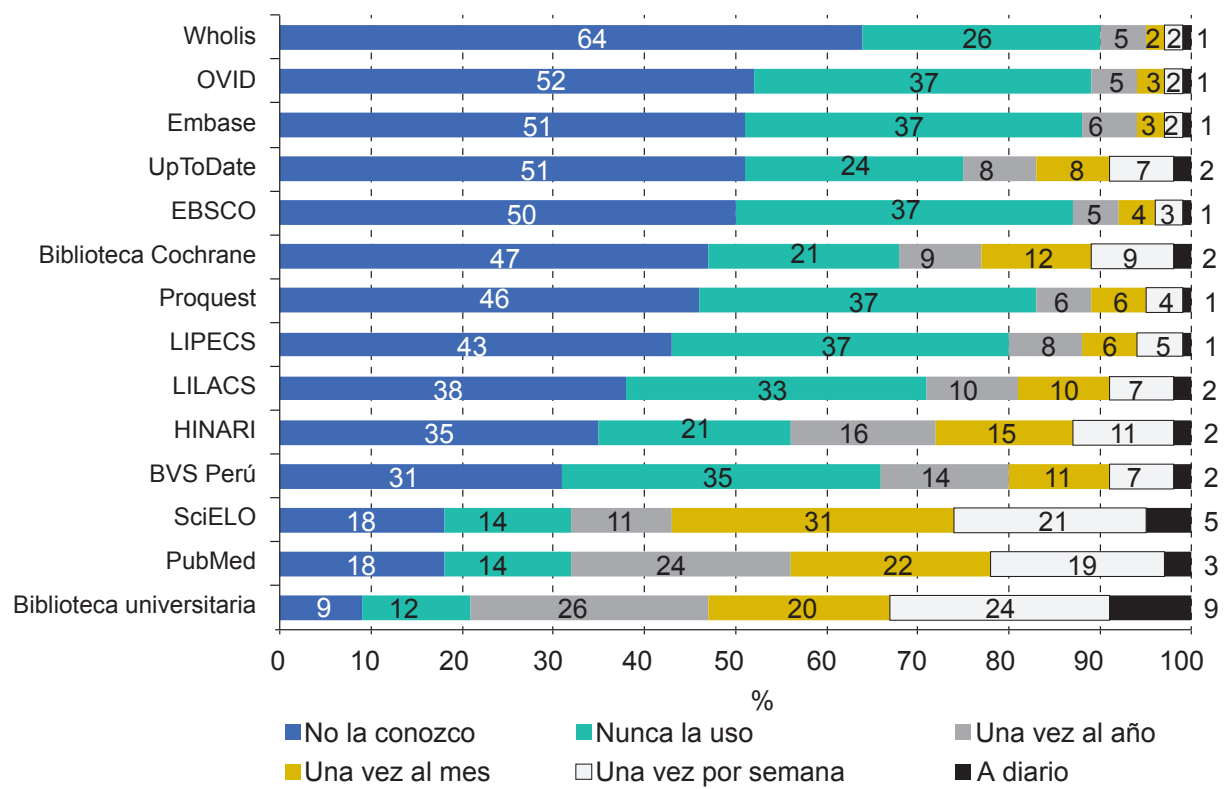

Figura 1. Uso de fuentes de información en estudiantes de Medicina de cuatro ciudades del Perú

Al indagar del uso de fuentes de la información, se encontró que existen algunas bases que aún no son conocidas por los estudiantes; las más resaltantes son: Wholis (64\%); OVID (52\%); Embase (51\%); UpToDate (51\%); EBSCO (50\%); Biblioteca Cochrane (47\%); Proquest (46\%); LIPECS (43\%); LILACS (38\%); HINARI (35\%) y BVS Perú (31\%). Las fuentes de información que son consultadas al menos una vez por semana son la biblioteca universitaria (33\%), SciELO (26\%), PubMed (22\%), HINARI (13\%) y la Biblioteca Cochrane (11\%) (Figura 1).

El 41,3\% (938) manifestaron que en el último año habían consultado una revista científica peruana, la más consultada fue la Revista Peruana de Medicina Experimental y Salud Pública (51,5\%). El 14,7\% (332) participaba de algún foro del área de Medicina, el más concurrido (30\%) fue el de la Sociedad Científica Médico Estudiantil Peruana (SOCIMEP).

Al realizar la estadística bivariada y multivariada para determinar los factores tecnológico-educativos que influyen en el uso regular de fuentes de la información, se encontró que el recibir una capacitación para el uso de dichas fuentes estuvo asociado al incremento de uso en todas las bases consultadas $(p<0,05)$, excepto para el caso de las consultas a la base SciELO $(p=0,053)$ y la biblioteca física universitaria $(p=0,509)$. Cuando se añadió el ajuste por el poseer una laptop/netbook, siguió sin presentar asociación a un uso frecuente en la base SciELO $(p=0,054)$ o la biblioteca universitaria $(p=0,503)$. Ajustado por las anteriores, más si el alumno poseía un smartphone, no se asoció a un uso frecuente de las fuentes de la información SciELO $(p=0,079)$, BVS Perú $(p=0,067)$ o de la biblioteca universitaria $(p=0,437)$. $\mathrm{Al}$ añadir al modelo anterior si el encuestado había realizado investigación científica, tampoco se asoció al uso frecuente de esas fuentes de la información; SciELO $(p=0,136)$, BVS Perú $(p=0,142)$ y la biblioteca universitaria $(p=0,487)$ (Tabla 3$)$.

\section{DISCUSIÓN}

En los resultados del presente estudio se encontró que aquellos estudiantes que habían recibido capacitación para el uso de fuentes de la información tuvieron mayor frecuencia de uso, hasta $19 \%$ en la base HINARI. $\mathrm{Si}$ es que se suman a esta capacitación el uso de TIC se mantiene la mejora en la gran mayoría de las bases consultadas (excepto en la biblioteca física, SciELO y BVS Perú). Esto es importante debido a que actualmente el acceso a Internet o el uso de TIC son medios necesarios para el desarrollo académico de estudiantes de Medicina ${ }^{(10)}$, permitiendo un mejor acceso electrónico a fuentes de información médica, mediante el uso de modernas tecnologías móviles ${ }^{(2,11)}$.

Si bien existieron diferencias entre cada sede, debido a la heterogeneidad de la oferta informática de cada ciudad (siendo esto tomado en cuenta al momento de hacer la estadística multivariada, al usar un ajuste para considerar a cada sede como un grupo independiente); la mayoría de estudiantes tenía mucho acceso a Internet y a la tecnologías de la comunicación e informática. Esto 
Tabla 3. Modelos bivariado y multivariado de uso de fuentes de información según factores educativos y uso de tecnología

\begin{tabular}{|c|c|c|c|c|c|c|}
\hline \multirow{2}{*}{$\begin{array}{l}\text { Base consultada cuando menos } \\
\text { una vez por semana }\end{array}$} & \multicolumn{2}{|c|}{ Fue capacitado $\mathrm{n}(\%)$} & \multicolumn{4}{|c|}{ Ajustado por (valor $p$ ) } \\
\hline & Sí & No & $\begin{array}{c}\text { A: } \\
\text { Capacitado }\end{array}$ & $\begin{array}{c}\text { B: } \\
\text { Tiene laptop* }\end{array}$ & $\begin{array}{c}\text { C: } \\
\text { Tiene smartphone ** }\end{array}$ & $\begin{array}{l}\text { Alumno } \\
\text { investiga \$ }\end{array}$ \\
\hline Biblioteca física & $211(35,7)$ & $533(32,3)$ & 0,509 & 0,503 & 0,437 & 0,487 \\
\hline PubMed & $197(33,3)$ & $296(17,9)$ & 0,001 & 0,001 & 0,002 & 0,006 \\
\hline Biblioteca Cochrane & $111(18,9)$ & $113(6,9)$ & $<0,001$ & $<0,001$ & $<0,001$ & 0,002 \\
\hline UpToDate & $93(15,8)$ & $91(5,5)$ & $<0,001$ & $<0,001$ & $<0,001$ & $<0,001$ \\
\hline HINARI & $157(26,6)$ & $128(7,8)$ & $<0,001$ & $<0,001$ & $<0,001$ & $<0,001$ \\
\hline SciELO & $207(35,0)$ & $376(22,7)$ & 0,053 & 0,054 & 0,079 & 0,136 \\
\hline LILACS & $102(17,4)$ & $99(6,0)$ & $<0,001$ & $<0,001$ & $<0,001$ & $<0,001$ \\
\hline LIPECS & $71(12,2)$ & $53(3,2)$ & $<0,001$ & $<0,001$ & $<0,001$ & $<0,001$ \\
\hline BVS Perú & $79(13,4)$ & $117(7,1)$ & 0,028 & 0,034 & 0,067 & 0,142 \\
\hline Proquest & $54(9,2)$ & $41(2,5)$ & $<0,001$ & $<0,001$ & $<0,001$ & $<0,001$ \\
\hline EBSCO & $43(7,4)$ & $26(1,6)$ & $<0,001$ & $<0,001$ & $<0,001$ & $<0,001$ \\
\hline OVID & $39(6,7)$ & $14(0,9)$ & $<0,001$ & $<0,001$ & $<0,001$ & $<0,001$ \\
\hline Embase & $34(5,8)$ & $14(0,9)$ & $<0,001$ & $<0,001$ & $<0,001$ & $<0,001$ \\
\hline Wholis & $28(4,8)$ & $25(1,5)$ & $<0,001$ & $<0,001$ & $<0,001$ & 0,001 \\
\hline
\end{tabular}

Valor $p$ obtenido mediante modelos lineales generalizados, familia Poisson, función de enlace log, con modelos robustos y con ajuste por cluster (sede de encuestado). ${ }^{*}$ Ajustado también por $A /{ }^{* *}$ Ajustado también por $A+B /{ }^{\$}$ Ajustado también por $A+B+C$.

puede generar una ventaja académica para la obtención de información actualizada. Existen reportes que esta tecnología puede ser incorporada con facilidad a la práctica diaria; en una revisión se encontró que el uso del asistente digital personal (PDA por sus siglas en inglés) es una herramienta valiosa para el personal y los estudiantes en el cuidado de la salud de los pacientes, debido a la necesidad de tener acceso a información actualizada en cualquier lugar y momento ${ }^{(12)}$.

Sin embargo, es preocupante saber que aún existen fuentes que son desconocidas para la mayoría de los estudiantes, como es el caso de Wholis, OVID, Embase y UpToDate; que menos del $50 \%$ las conozcan podría deberse a que sus casas de estudio no han conseguido las licencias para su uso o que, a pesar de tenerlas, los estudiantes no las usan. Las fuentes más usadas siguen siendo PubMed, SciELO y la biblioteca universitaria. Este hallazgo es similar al encontrado en un país africano en vía de desarrollo, que mostró que los conocimientos eran inadecuados y la utilización de las TIC eran pobres en el personal médico ${ }^{(13)}$. En nuestro medio, las universidades son las encargadas de generar estrategias para la mejora de esta situación, pero hace algún tiempo ciertas entidades nacionales también han contribuido, como es el caso del Consejo Nacional de Ciencia, Tecnología e Innovación Tecnológica (CONCYTEC), que no solo ha conseguido importantes bases de datos científicas, sino que capacita de manera presencial y a distancia a los estudiantes y profesionales interesados (http://alicia.concytec.gob.pe/vufind/).

Se encontró que la capacitación fue un importante factor asociado para un uso más frecuente de la gran mayoría de las bases consultadas. Según estudios similares en estudiantes e internos de Medicina, el realizar estrategias innovadoras sobre búsqueda bibliográfica y lectura crítica mejoran los indicadores del uso de bases de datos de investigación científica ${ }^{(14)}$. De esta manera, se adquiere un adecuado uso y fácil acceso no solo en los estudiantes, sino también en los docentes de investigación y otro personal de salud ${ }^{(15,16)}$. Solo las más conocidas (SciELO y la biblioteca física) no mostraron una mejora significativa, esto se podría explicar por su fácil uso o mayor difusión en nuestro medio, que lo diferencia de otras bases que requieren una capacitación específica $u$ otros factores que predispongan su mayor uso ${ }^{(17,18)}$.

En el presente estudio el smartphone fue un recurso usado por la mayoría de la población, que se asoció al uso frecuente de fuentes de la información en once de las catorce bases consultadas, esto se puede explicar porque las TIC disponibles en ordenadores y dispositivos móviles contribuyen potencialmente al acceso de información e intercambio de nuevos conocimientos ${ }^{(19)}$, pues ofrecen diversas herramientas para la investigación y la educación; siendo útiles en la práctica asistencial habitual, en la cibermedicina y la telemedicina ${ }^{(20)}$. El uso de dispositivos móviles ha obtenido mayor relevancia en la educación y la práctica médica ${ }^{(21)}$, pues permiten una mayor eficacia en la búsqueda de información ${ }^{(22,23)}$, y son los médicos y los estudiantes de Medicina quienes vienen adoptando este tipo de tecnología ${ }^{(21)}$, mejorando así la toma de decisiones en la práctica diaria. Al no indagar más acerca de las características de este uso, esto debería ser motivo de futuras investigaciones, para determinar si es que usan y conocen las herramientas 
ligadas a la práctica médica que poseen este tipo de dispositivos ${ }^{(24)}$.

Por último, muy pocos de los encuestados habían realizado una investigación científica, sin embargo, al ajustar por esta variable se mantuvo la asociación de aumento del uso en once del total de bases consultadas. Esto se puede deber a que la práctica continua del proceso de investigación brinda experiencia en el transcurso de obtener antecedentes para el trabajo que se realizará; ya que para efectuar esto se deberán obtener referencias bibliográficas actualizadas y de buena calidad, para lo cual es necesario el uso de las TIC y fuentes de información confiables ${ }^{(25-27)}$. Es por eso que la investigación siempre será una estrategia que adopten las universidades para la mejora continua y la generación de conocimientos, debiendo ser realizada de manera idónea por la plana docente capacitada y los círculos de investigación de nuestras casas de estudio ${ }^{(28-29)}$.

Se tuvo la limitación de que los resultados obtenidos reflejan la realidad de solo cuatro ciudades, más no de todos los estudiantes de Medicina del Perú, no se pudo realizar un muestreo debido a la política de reserva de los datos de las universidades, sin embargo, debido a que se sabía que los encuestados podían tener diferencias por las características de sus centros de estudio, se hizo la estadística multivariada ajustando a cada sede como un grupo independiente; no obstante, este estudio refleja el uso de base de datos en cuatro de las ciudades más importantes y grandes del país, por lo que esto puede ser una buena aproximación a la realidad. Además, no se evaluaron otras características del proceso de búsqueda de información científica (estrategias de búsqueda, calidad y discriminación de la información encontrada, uso de metabuscadores, características de la capacitación), esto por no haber sido el objetivo del estudio.

Se concluye que la capacitación y el uso de TIC estuvieron asociados al uso más frecuente de las fuentes de información científica en estudiantes de Medicina de cuatro ciudades del Perú. Estos resultados pueden ser usados de referencia para que las instituciones educativas puedan evaluar su realidad y generar estrategias de capacitación en el uso de las fuentes de información, para así ayudar a la capacitación y la mejora continua en el pre y posgrado.

Contribuciones de autoría: CRM, ALR y MVG tuvieron la idea de investigación y diseñaron el estudio, DVG, LTU, MVE y MAC recopilaron los datos, CRM y MVG realizaron el análisis e interpretación de datos, todos los autores participaron en la redacción del manuscrito y aprobaron la versión final a publicar.

Miembros de la Red GIS Peru: Noé Atamari-Anahui (Universidad Nacional de San Antonio Abad del Cusco, Perú), Dante CondoriMerma (Universidad Nacional de San Agustín de Arequipa, Perú), Lisbeth R. Mamani-Humpiri (Universidad Ricardo Palma, Perú).

Fuentes de financiamiento: autofinanciado.

Conflictos de interés: los autores declaran no tener conflictos de interés.

\section{REFERENCIAS BIBLIOGRÁFICAS}

1. L'Etang H. Effectiveness and efficiency. Random reflections on health services. J R Soc Med. 1990 Sep; 83(9):601-2.

2. Canelo C, Alarcon J, Amao E, Beteta V, Monge E. Conocimientos, actitudes y prácticas de la medicina basada en evidencias en médicos asistentes y residentes en dos hospitales de Lima-Perú. Rev Medica Hered. 2007;18(2):76-84.

3. Veloz-Martínez MG, Almanza-Velasco E, Uribe-Ravell JA, González LL-D, Quintana-Romero V, Alanís-López P. Uso de tecnologías en información y comunicación por médicos residentes de ginecología y obstetricia. Inv Ed Med. 2012;1(4):183-9.

4. Agámez $\mathrm{S}$, Aldana $\mathrm{M}$, Barreto $\mathrm{V}$, Santana A, Caballero-Uribe C. Aplicación de las nuevas tecnologías de la Información en la Enseñanza de la Medicina. Salud Uninorte. 2009;25(1):150-71.
5. De Fernández ICR, Teppa $S$, Fernández MC. Las TIC en el desarrollo de competencias en estudiantes del Programa de Medicina. UCLA. Educare. 2011;14(1):5-27.

6. Andalia RC, Franco NMP, Rodríguez KP, Castells MM. Propuesta de recursos de información en salud útiles a estudiantes de la carrera de Medicina [internet]. Correo Científico Méd 2012;16(3) [citado 9 de febrero 2015]. Disponible en: http://www. medigraphic.com/pdfs/correo/ccm2012/ccm123s.pdf

7. Custodio-Marroquín J, FernándezOtoya L. Uso de las Tecnologías de Información y Comunicación en Médicos de la Región Lambayeque. Rev. Cuerpo Méd. HNAAA. 2013;6(1):60-1.

8. Herrera-López V, StucchiPortocarrero S, Vega-Galdós F. Encuesta virtual sobre uso de
Internet y medios informáticos entre psiquiatras y residentes de psiquiatría en el Perú. Rev Neuropsiquiatr. 2013;74(1):191-9.

9. Lara K, Miyahira J. Uso de internet por estudiantes del internado de medicina en Lima Metropolitana. Rev Medica Hered. 2009;20(3):156-61.

10. Batten LM, Chowdhury M, Drew J. The major drawbacks to the use of wireless communication products in education. 1st Australian Information Security Management Conference. Perth, Western Australia. School of Information and Computer Management (SCISSEC research group), Edith Cowan University. 24 November 2003.

11. Walton G, Childs S, Blenkinsopp E. Using mobile technologies to give health students access to learning resources in the UK community setting. Health Info Libr J. 2005 Dec;22 Suppl 2:51-65. 
12. Lindquist AM, Johansson PE, Petersson GI, Saveman BI, Nilsson GC. The use of the Personal Digital Assistant (PDA) among personnel and students in health care: a review. J Med Internet Res. 2008 Oct 28;10(4):e31. doi: 10.2196/jmir.1038.

13. Woreta SA, Kebede Y, Zegeye DT. Knowledge and utilization of information communication technology (ICT) among health science students at the University of Gondar, North Western Ethiopia. BMC Med Inform Decis Mak. 2013 Mar 3;13:31. doi: 10.1186/14726947-13-31.

14. Skinner H, Biscope S, Poland B. Quality of internet access: barrier behind internet use statistics. Soc Sci Med. 2003 Sep;57(5):875-80.

15. Huamani-Navarro M, AlegríaDelgado D, López-Sánchez $M$, Tarqui-Mamani CB, OrmeñoCaisafana L. Conocimientos, prácticas y habilidades sobre la búsqueda bibliográfica y percepción estudiantil sobre la capacitación universitaria en investigación, en estudiantes de obstetricia. Educ Médica. 2011;14(4):235-40.

16. Mejia CR, Caceres OJ, Vera CA, Nizama-Vía A, Curioso WH, MaytaTristán $\mathrm{P}$, et al. Uso de fuentes de información en médicos recién graduados de Lima. Rev Peru Med Exp Salud Publica. 2014;31(4):716-20.

17. Romanov K, Aarnio M. A survey of the use of electronic scientific information resources among medical and dental students. BMC Med Educ. 2006 May 9;6:28.

18. Giglia E. Beyond PubMed. Other free-access biomedical databases. Eura Medicophys. 2007 Dec;43(4):563-9.

19. Bullock A. Does technology help doctors to access, use and share knowledge? Med Educ. 2014 Jan;48(1):28-33. doi: 10.1111/ medu. 12378 .

20. Viloria C. Information technology to education, research and application in health. Benefits and challenges. Rev Salud Uninorte. 2009;25(2):331-49.

21. Boruff JT, Storie D. Mobile devices in medicine: a survey of how medical students, residents, and faculty use smartphones and other mobile devices to find information. J Med Libr Assoc. 2014 Jan;102(1):22-30. doi: 10.3163/1536-5050.102.1.006.

22. Mickan S, Tilson JK, Atherton $\mathrm{H}$, Roberts NW, Heneghan C. Evidence of effectiveness of health care professionals using handheld computers: A scoping review of systematic reviews. J Med Internet Res. 2013;15(10):e212.

23. Kho A, Henderson LE, Dressler DD, Kripalani S. Use of handheld computers in medical education. A systematic review. J Gen Intern Med. 2006 May;21(5):531-7.

24. Ozdalga E, Ozdalga A, Ahuja N. The Smartphone in medicine: a review of current and potential use among physicians and students. J Med Internet Res. 2012 Sep 27;14(5):e128.
25. Estrada JM. Capítulo 2: La búsqueda bibliográfica y su aplicación en PubMed-MedLine. Semergen-Med Fam. 2007;33(4):193-9.

26. Peña JLM. Tecnologías de la información y comunicaciones. Educ Médica. 2004;7 suppl.1:15-22.

27. Pestaña A. El Medline como fuente de información bibliométrica de la producción española en biomedicina y ciencias médicas: comparación con el Science Citation Index. Med Clin (Barc). 1997,109: 506-11.

28. Pereyra-Elías R, Huaccho-Rojas JJ, Taype-Rondan A, Mejia CR, MaytaTristán P. Publicación y factores asociados en docentes universitarios de investigación científica de escuelas de Medicina del Perú. Rev Peru Med Exp Salud Publica. 2014;31(3):424-30.

29. Taype-Rondán Á, Huaccho-Rojas J, Pereyra-Elías R, Mejia CR, MaytaTristán P. Características de los cursos de investigación en escuelas de Medicina del Perú. Arch Med. 2015;11(2):e1. doi: 10.3823/1243.

Correspondencia: Christian R. Mejia.

Dirección: Av. Las Palmeras 5713 - Los Olivos. Lima 39 - Perú.

Teléfono: (511) 997643516

Correo electrónico: christian.mejia.md@ gmail.com

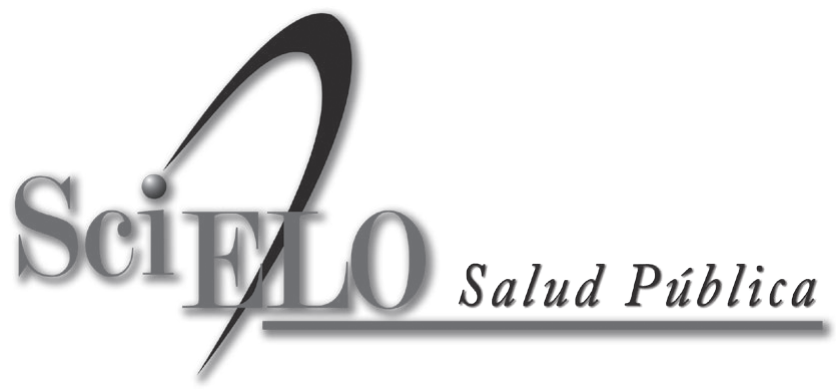

